\title{
VIOLENCIAS CONTRA LAS MUJERES EN EL CONTEXTO DE LA COVID-19: ESCENARIOS Y DESAFÍOS
}

VIOLENCE AGAINST WOMEN IN MEXICO AMIDST COVID-19: SCENARIOS AND CHALLENGES

Lorena Vázquez Correa

lorena.vazquez@senado.gob.mx 
Para citar este artículo:

Vázquez Correa, L. (2020). Violencias contra las mujeres en el contexto de la COVID-19 escenarios y desafíos. ESPACIO I+D, INNOVACIÓN MÁS DESARROLLO, 9(25). https://doi. org/10.31644/IMASD.25.2020.a08

\section{RESUMEN}

En este artículo se analiza el incremento de las violencias contra las mujeres en los hogares durante la pandemia de la COVID-19 y las respuestas institucionales del Estado mexicano, así como el trabajo de las organizaciones de la sociedad civil respecto de las acciones que se dan tanto en la esfera pública como en la privada para prevenir, atender, sancionar y erradicar la violencia contra las mujeres y para garantizar el respeto de sus derechos humanos. Se argumenta que tener un enfoque de género en las políticas de mitigación de la crisis implica, como una condición necesaria, que el Estado reconozca que la violencia contra las mujeres es la pandemia más letal contra este sector de la población y que, por tanto, los programas de atención a estos grupos vulnerables deben catalogarse como prioritarios en las finanzas y estrategias de seguridad pública.

\section{Palabras clave}

Violencia contra las mujeres; COVID- I9; feminicidio; pandemia; violencia familiar. 


\section{- Abstract-}

This essay analyzes the increase in violence against women in home during the COVID-19 pandemic and the institutional responses of the Mexican State, and the work of civil society organizations regarding the actions that take place both in the public as well as in the private sphere to prevent, attend to, punish and eradicate violence against women and to guarantee respect for their human rights. It is argued that having a gender approach in crisis mitigation policies implies, as a necessary condition, that the State recognize that violence against women is the most lethal pandemic against this sector of the population and that, therefore, care programs for these vulnerable groups should be classified as a priority in finance and public security strategies.

\section{Keywords}

Violence Against Women; COVID- I 9; femicide; pandemic; family violence. 
a emergencia sanitaria derivada de la COVID-19, está provocando impactos graves en todas las dimensiones sociales y, específicamente, en la vida de las mujeres y niñas, la pandemia ha tenido impactos diferenciados (OEA, 2020; ONU, 2020; Inmujeres, 2020; Intersecta et al, 2020). De acuerdo con la Organización de las Naciones Unidas, el confinamiento sanitario aviva la tensión y el estrés generados por preocupaciones relacionadas con la salud, el dinero y el trabajo, al tiempo que refuerza el aislamiento de quienes ya son víctimas de violencia, separándolas de las personas, recursos e instituciones para defenderse. Si a ello le sumamos las masculinidades tóxicas y conductas machistas que persisten en las sociedades, se propicia un ambiente de mayor vulnerabilidad para las mujeres y niñas frente a expresiones violentas en el hogar.

Así, al tiempo que los sistemas sanitarios se esfuerzan al límite para atender a pacientes de la pandemia, los refugios para las víctimas de violencia familiar alcanzan también su máxima capacidad, mientras que las instituciones gubernamentales $y$ de justicia suspendieron temporalmente funciones jurisdiccionales y administrativas, incluyendo las órdenes de protección para las mujeres, los divorcios, los juicios por pensiones alimentarias y las denuncias penales. Incluso, algunas instituciones de atención a mujeres víctimas de violencia tuvieron recortes, incertidumbre y hasta la cancelación de su presupuesto, lo que dificultó aún más, una respuesta eficaz a la problemática (Intersecta, et al, 2020).

En el marco de estas consideraciones, en este artículo se analiza el incremento de las violencias contra las mujeres en los hogares durante la pandemia del COVID-19 y las respuestas institucionales del Estado mexicano, así como el trabajo de las organizaciones de la sociedad civil respecto de las acciones que se dan tanto en la esfera pública como en la privada para prevenir, atender, sancionar y erradicar la violencia contra las mujeres y para garantizar el respeto de sus derechos humanos. Se argumenta que tener un enfoque de género en las políticas de mitigación de la crisis implica, como una condición necesaria, que el Estado reconozca que la violencia contra las mujeres es la pandemia más letal contra este sector de la población y que, por tanto, los programas de atención a estos grupos vulnerables deben catalogarse como prioritarios en las finanzas y estrategias de seguridad pública.

\section{REFERENTES CONCEPTUALES}

La violencia contra las mujeres es un problema público preocupante. Sin embargo, pese a la implementación de políticas públicas para garantizar la seguridad, la integridad, la dignidad y la vida de las mujeres, la problemática ha incrementado, desde antes y durante la pandemia de COVID-19, 
convirtiéndose así en el principal obstáculo para su desarrollo y participación en la vida económica, política, social y cultural del país.

De acuerdo con la Organización de las Naciones Unidas, la violencia contra las mujeres se define como el conjunto de expresiones, acciones u omisiones "basado en la pertenencia al sexo femenino que tenga o pueda tener como resultado un daño o sufrimiento físico, sexual o psicológico para la mujer, así como las amenazas de tales actos, la coacción o la privación arbitraria de la libertad, tanto si se producen en la vida pública como en la vida privada" (ONU, 2010 ; Resolución 48/I 04; Recomendación General 19 de la CEDAW).

En ocasiones los términos violencia basada en género y violencia contra las mujeres son usados indistintamente en la literatura, sin embargo, son distintos (ONU, 20I0). La violencia basada en género refiere a aquellas expresiones dirigidas contra una persona en razón del género que él o ella tiene, así como por las expectativas sobre el rol que él o ella deba cumplir en una sociedad o cultura. De ahí que, tanto hombres como niños también pueden ser víctimas de la violencia basada en el género, especialmente de la violencia sexual. Por su parte, el término violencia contra las mujeres visibiliza el fenómeno de que las expresiones de violencia de género afectan desproporcionadamente a las mujeres y niñas, es decir, afectan en mayor medida a este sector de la población (ONU, 20l0).Aunado a lo anterior, es importante advertir que, en las convenciones internacionales el término mujeres comprende a mujeres de todas las edades, incluidas las niñas (ONU, 2006).

Un elemento a considerar es que las manifestaciones de violencia contra las mujeres pueden llevarse a cabo en el ámbito público o en el ámbito privado. Tradicionalmente, el espacio público se concibió como el espacio "de los ciudadanos, de la relación entre pares hombres (sic.) y [...] el de la presencia del Estado, donde los derechos humanos protegerían de cualquier abuso" (Fríes y Hurtado, 20 I0: I3). Mientras que el privado fue ligado "a la familia, entidad natural en la que mujeres tienen un mismo estatus, el de desiguales frente al jefe de familia" (Fríes y Hurtado, 20 I0: I3, citado en CNDH, 2016).

Actualmente, ambas esferas de manifestación de las violencias -pública y privada-conllevan obligaciones a los Estados para participar y emprender acciones en la lucha por la erradicación de la violencia contra las mujeres. Dicho reconocimiento deriva de los esfuerzos de la sociedad civil organizada y los gobiernos que se han traducido en instrumentos internacionales para el reconocimiento de la responsabilidad del Estado frente a las violaciones a los derechos humanos de las mujeres, en razón de la histórica discriminación que han vivido (CNDH, 2016).

La literatura distingue entre tipos y modalidades de violencias $(\mathrm{CNDH}$, 20 16; Fríes y Hurtado 2010; LGAMVLV, 2020). Los primeros refieren al 
conjunto de acciones u omisiones que lesionen -o sean susceptibles de dañar- la dignidad o libertad de las mujeres, tales como la psicológica, física, emocional, patrimonial, económica, sexual u otras. De ahí que, los tipos de violencia pueden presentarse de manera combinada con otros y puede manifestarse en distintos espacios de interacción entre hombres y mujeres. Por su parte, las modalidades refieren a los distintos ambientes o modos en que las personas interactúan y donde pueden llevarse a cabo las expresiones de violencia, tales como el espacio familiar, laboral, institucional, político u otros. Así, en las modalidades pueden concurrir varios tipos de violencias y dichas expresiones pueden presentarse tanto en la esfera pública, como en la privada.

\section{Tabla I}

Esferas, tipos y modalidades de violencia contra las mujeres

\begin{tabular}{|c|c|c|}
\hline Esfera & Tipos & Modalidades \\
\hline Privada & Psicológica & Familiar \\
\hline \multirow[t]{5}{*}{ Pública } & Física & Laboral y Docente \\
\hline & Patrimonial & Comunitaria \\
\hline & Económica & Institucional \\
\hline & Sexual & Política \\
\hline & $\begin{array}{l}\text { Cualesquiera otras violencias que } \\
\text { lesionen - o sean susceptibles de dañar- } \\
\text { la dignidad o libertad de las mujeres }\end{array}$ & Feminicida \\
\hline
\end{tabular}

\section{VIOLENCIA CONTRA LAS MUJERES: LA OTRA PANDEMIA}

La violencia contra las mujeres es un tema de emergencia nacional, de salud pública, de derechos humanos y de justicia, que se ha profundizado, aún más, en el contexto de la pandemia de la COVID-I 9 (Intersecta, et al, 2020). En los países de América Latina se han incrementado los reportes de violencias contra las mujeres de manera paralela a la implementación de medidas de confinamiento en los hogares para reducir la propagación del virus, y México no es la excepción (ONU Mujeres 2020; OEA 2020).

De acuerdo con los reportes mensuales del Secretariado Ejecutivo del Sistema Nacional de Seguridad Pública (SESNSP) de enero a julio 2020 se registraron un total de 716,337 llamadas de emergencia relacionadas con violencia contra la mujer $(154,610)$, violencia sexual $(9,983)$ ', incidentes de violencia de

1 En particular, las cifras son las siguientes: violencia contra la mujer $(154,610)$, violencia sexual $(9,983$, en total lo que incluye abuso sexual con 3,026$)$, acoso u hostigamiento sexual $(4,777)$ e incidentes de violación $(2,180)$, incidentes de violencia de pareja $(138,832)$ y violencia familiar $(412,912)$. 
pareja $(\mid 38,832)$ y violencia familiar $(4|2,9| 2)$. Lo que significó aproximadamente un promedio de 142 llamadas por hora (SESNSP-CNI, 2020). ${ }^{2}$

Las cifras de homicidios dolosos de mujeres y los feminicidios también incrementaron y son alarmantes. De acuerdo con el Secretariado Ejecutivo del Sistema Nacional de Seguridad Pública, de enero a julio de 2020 se registraron 2,223 víctimas mujeres a nivel nacional (549 feminicidios y I,674 homicidios dolosos), en promedio once por día (SESNSP, 2020: I329). Aunado a lo anterior, al comparar las cifras de marzo de 2020 y abril de 2020 se muestra que los asesinatos de mujeres aumentaron en un 2 por ciento, mientras que los de los hombres se redujeron un 0.2 por ciento (Intersecta, et al, 2020).

Los presuntos delitos de violencia de género en todas sus modalidades tuvieron su pico más alto en julio de 2020 (367 casos) y, en promedio incrementó 25 por ciento la incidencia de este delito comparado con los primeros siete meses de años anteriores (SESNSP, 2020). Por su parte, la violencia familiar registró en marzo de 2020 su pico más alto de los últimos seis años (20,504 casos) y la tendencia nacional de los primeros siete meses del año muestra un incremento del 1.6 por ciento respecto de los mismos periodos en años anteriores.

Las entidades con las cifras más altas de delitos de violación son Estado de México, Ciudad de México, Nuevo León y Chihuahua y, lamentablemente, en dichos casos el agresor suele ser muy cercano a la familia o incluso parte de ella. En ocasiones el agresor ha sido el vecino, pero en otros el padrastro, el abuelo, el tío e incluso el propio padre (ONU Mujeres 2020; OEA 2020). En suma, estas cifras reflejan que en el seno familiar es el ámbito donde se manifiesta un gran porcentaje de las violencias contra las mujeres e infantes (Intersecta, et al, 2020).

Más allá de las cifras oficiales, organizaciones de la sociedad civil como la Red Nacional de Refugios A.C. (RNR) ${ }^{3}$ reportan que desde marzo (mes en que comenzó el confinamiento en México) han atendido a 14,599 mujeres, niñas y niños víctimas de violencia, cifra que representa un incremento de 81 por ciento respecto del periodo de marzo a junio de 2019 (Intersecta, et al, 2020). Asimismo, las solicitudes de apoyo vía telefónica y por redes sociales han tenido un incremento de 7 I por ciento, comparando este

Las llamadas de emergencia no son denuncias ante una autoridad, se trata únicamente de probables incidentes de emergencia, con base en la percepción de la persona que realiza la llamada.

La Red Nacional de Refugios, A.C. está integrada por 69 espacios de prevención, atención y protección para mujeres, niños y niñas que viven violencia. La Red cuenta con 31 Refugios, 29 Centros de Atención Externa, 5 Casas de Emergencia y 4 Casas de Transición, que, en conjunto, pueden encontrarse en las 32 entidades federativas del país. Además, brindan orientación y atención por vía telefónica y por redes sociales. 
periodo con el mismo del año pasado. Así, la RNR reportó haber recibido 6,475 llamadas o mensajes de auxilio, de las cuales 74 por ciento fueron de mujeres, 16 por ciento de instancias de gobierno y 10 por ciento de hombres.

Del total de llamadas, 46 por ciento se concentraron en la Ciudad de México y el Estado de México, seguidos de Chiapas, Coahuila, Hidalgo, Michoacán, Morelos, Puebla, Sinaloa, Guanajuato y Veracruz (SESNSP, 2020). Asimismo, la Red informó que 46 por ciento de las hijas e hijos de las mujeres que pidieron apoyo, fueron víctimas de violencias en casa durante el confinamiento, y que más de 500 niñas, niños y adolescentes que ingresaron a un refugio, sufrían violencia sexual durante el confinamiento sanitario (Intersecta, et al, 2020).

De acuerdo con el Reporte de la Red Nacional de Refugios, los tipos de violencias que mayoritariamente se han perpetrado fueron: violencia física (4I.5\%), emocional (23.29\%), económica y patrimonial $(5.46 \%)$ y sexual (3.63\%).Además, la Red reportó que dos de cada 10 mujeres que le solicitaron apoyo, refirieron haber acudido con anterioridad a otra instancia estatal o municipal, sin recibir la ayuda esperada (Fríes y Hurtado, 2010).

Incluso antes de que existiera la COVID-19, la violencia contra las mujeres y niñas en el espacio privado era una de las violaciones de los derechos humanos más flagrantes $y$, con el avance de la pandemia, el problema creció con múltiples efectos en el bienestar de las mujeres, su salud sexual y reproductiva, su salud mental y su capacidad de liderar y participar en la recuperación de las sociedades y economías.

Los impactos diferenciados de la pandemia también se exacerban en la esfera pública y, en particular para las sobrevivientes de violencia, el impacto económico de la pandemia puede generar barreras adicionales para dejar a una pareja violenta (ONU Mujeres, 2020; OEA, 2020). Asimismo, las dificultades para acceder a métodos anticonceptivos por el confinamiento, tendrán como consecuencia el incremento de embarazos no deseados.

La Organización de los Estados Americanos estima que en los países de la región, 18 millones de mujeres adicionales perderán su acceso a anticonceptivos modernos, dado el contexto actual de las pandemias de COVID-19, lo cual podría incrementar la mortalidad y morbilidad materna, así como aumentar las tasas de embarazo adolescente, $\mathrm{VIH}$ y otras infecciones de transmisión sexual (OEA, 2020: 18-19).

En México, la Secretaría de Salud y el Consejo Nacional de Población preveen alrededor de 145 mil embarazos excedentes, de los cuales aproximadamente 21 mil serán de adolescentes menores de 19 años, esto es, 20 por ciento más de lo reportado en el mismo periodo del año anterior (Toche, 2020; Inmujeres, 2020). Al respecto, es importante considerar que el embarazo de 10 a 14 años, mínimo, no solo es un asunto de salud pública, o económico, sino un asunto de seguridad y justicia, pues constituye el 
resultado de un hecho forzado, impuesto, producto de la violencia sexual y que, por tanto, requiere de estrategias de protección por parte del Estado.

De acuerdo con la metodología para estimar el impacto socioeconómico del embarazo y la maternidad en adolescentes de América Latina y el Caribe, denominado MILENA, en México, los ingresos anuales de las mujeres que han tenido hijos siendo adolescentes son casi 32 por ciento menores a los de quienes fueron madres en edad adulta. El embarazo adolescente representa un costo total de casi 63,000 millones de pesos mexicanos anuales, lo que equivale al $0.27 \%$ del Producto Interno Bruto, con lo cual se podrían construir y equipar 2I hospitales de tercer nivel, edificar 9,582 escuelas o 4, 233 kilómetros de carreteras rurales (Toche, 2020).

Peor aún, la Organización de los Estados Americanos estima que, fuera de los hogares, las mujeres también constituyen el mayor contingente que está asumiendo los cuidados en el sector de la sanidad, en el trabajo doméstico remunerado y en centros especializados de cuidado de menores, adultos/as mayores y personas con discapacidad, situación que conlleva impactos diferenciados sobre su salud y las expone a un mayor riesgo de contagio (OEA, 2020).

Frente a este escenario, diversas organizaciones sociales, políticas, nacionales e internacionales se han sumado al llamado para incluir a las mujeres en la toma de decisiones, así como implementar políticas con enfoque de género para la gestión, mitigación y recuperación de la crisis económica, sanitaria y de seguridad pública (Intersecta et al., 2020).

\section{POLÍTICAS PÚBLICAS SIN ENFOQUE DE GÉNERO: LAS RESPUESTAS INSTITUCIONALES}

Las políticas públicas y las respuestas institucionales a la violencia contra las mujeres en México no han sido suficientes para prevenir, atender, sancionar y mitigar la violencia contra las mujeres, ni antes ni durante la pandemia de COVID-19 (Intersecta, et al, 2020). La falta de coordinación en los servicios disponibles en los poderes judiciales, el acceso a la información sobre los servicios disponibles para las víctimas, la austeridad presupuestaria sin perspectiva de género y la reducción e incertidumbre presupuestaria de los refugios de víctimas, son algunos ejemplos de la falta de estrategias y políticas del Estado que contribuyan a hacer frente a la violencia contra las mujeres, no solo durante la pandemia, sino desde antes.

En los casos de violencias contra las mujeres existen dos escenarios: aquellos en los que las víctimas denuncian y aquellos en los que no. Dicho planteamiento es importante porque de ello dependerá la implementación de las políticas públicas. Según la Encuesta Nacional sobre la Dinámica de las Relaciones en los Hogares, una de cada cuatro mujeres mayores de I5 
años ha experimentado agresiones físicas o sexuales de su pareja a lo largo de la relación. 89 por ciento de las mujeres que pasa por este trance no presentan una queja o denuncia ante una autoridad. De ese total de mujeres que no denunciaron ni solicitaron ayuda, 20 por ciento se quedó callada por miedo a las consecuencias y el 25 por ciento porque no sabía cómo o dónde denunciar, desconfiaba de las autoridades o ignoraba que existían leyes para sancionar la violencia en el hogar (Endireh, 20l6).

Frente a ello, Intersecta, Equis: Justicia para las mujeres y la Red Nacional de Refugios, han propuesto diseñar e implementar estrategias desde la educación básica, que tengan como objetivo educar para la igualdad entre hombres y mujeres en ambientes libres de violencias, tanto en el espacio público como en el privado; que informen a las mujeres sobre sus derechos humanos, que la violencia familiar no es normal, pero también que atiendan y combatan las masculinidades tóxicas y conductas machistas que permean a las sociedades y constituyen un obstáculo para alcanzar la igualdad sustantiva entre mujeres y hombres (Intersecta, et al, 2020).

En el caso del II por ciento de las mujeres que denuncian o piden ayuda, se ha identificado que "cuando una mujer denuncia es porque ya está en riesgo su vida" (Sandoval, 2020). En este tenor, el Secretariado Ejecutivo del Sistema Nacional de Seguridad Pública registró la tasa más alta en los últimos seis años de denuncias por violencia familiar, 190.9 por cada 100 mil mujeres, entre enero y julio de 2020, respecto del mismo periodo de los años anteriores, pese a la implementación de medidas de confinamiento y la suspensión temporal (o restringida) de los servicios de las fiscalías (Cerillo, 2020).

En los casos donde hay denuncias, diversas organizaciones de la sociedad civil han sugerido que la vía de acción consiste en diseñar e implementar políticas en las que se proteja de manera inmediata a la víctima de violencia que se atreven a denunciar, que les brinde un espacio de apoyo integral psicológico, legal, de salud, y que proteja su integridad física (Intersecta, et al, 2020). También es relevante fortalecer el entramado institucional que atiende a las víctimas de violencia (fiscalías, ministerios públicos, entre otras), así como dotarlas de presupuesto programado y recursos humanos especializados para atender, prevenir y garantizar la integridad de las mujeres y el acceso a la justicia, a fin de combatir la impunidad del delito.

Finalmente, el análisis de los impactos diferenciados y desproporcionados que la pandemia tiene en la vida de las mujeres, implica considerar que las violencias contra las mujeres son consecuencia de una cultura machista y patriarcal, que incluso puede llegar a ser mortal, como ocurre con los feminicidios. Frente a esta realidad, negar o ignorar el incremento de las violencias contra las mujeres durante la pandemia de COVID-19, así como recortar los recursos institucionales para atender el fenómeno es omisión 
y negligencia en el servicio público, constituye violencia institucional y contribuye al incremento y la impunidad de las conductas machistas que atentan contra los derechos humanos de las mujeres.

\section{EL PAPEL DEL ESTADO MEXICANO FRENTE AL INCREMENTO DE LAS VIOLENCIAS DURANTE LA PANDEMIA}

Las respuestas instruccionales al incremento de las violencias contra las mujeres en el contexto de la pandemia han sido insuficientes. Respecto de la atención inmediata a las víctimas, la Red Nacional de Refugios, Equis: Justicia para las mujeres e Intersecta, documentaron que los recursos y programas públicos con enfoque de género, habían sufrido afectaciones importantes, derivadas de las políticas de la administración federal 2018-2024, las cuales consistieron en: I) la negativa por parte del Ejecutivo de trabajar con las organizaciones de la sociedad civil, 2) al interés de reducir intermediarios y priorizar las transferencias directas a personas beneficiarias (debilitamiento de los refugios para mujeres) y, 3) a la llamada "austeridad republicana" que adoptó el Gobierno Federal, lo que derivó en recortes presupuestales a los programas para prevenir y atender las violencias contra las mujeres (Intersecta, et al, 2020: 26). ${ }^{4}$

Entre las medidas de austeridad implementadas se encontró el recorte de 75 por ciento del presupuesto disponible para servicios generales y materiales y suministros del Instituto Nacional de los Pueblos Indígenas, lo que se tradujo en la cancelación de recursos a las Casas de la Mujer Indígena, sin indicación expresa de cuándo se retomará la asignación de recursos (Intersecta et al., 2020: 32-33). Con ello, se pone en riesgo el único programa del país que atiende a mujeres indígenas en sus derechos sexuales y reproductivos, que previene la violencia de género mediante la difusión de información y que brinda servicios de traducción y acompañamiento a las víctimas de violencia en las comunidades indígenas.

En relación con la respuesta de las instituciones de justicia, que atienden a las víctimas de violencia, la organización Equis: Justicia para las Mujeres documentó que 87 por ciento de los órganos jurisdiccionales del Poder Judicial mexicano reprobaron la evaluación sobre la atención institucional de las necesidades de las mujeres víctimas de violencia en sus planes de suspensión de labores derivados de la pandemia, es decir que herramientas

$4 \quad$ Cf. Decreto por el que se establecen las medidas de austeridad que deberán observar las dependencias y entidades de la Administración Pública Federal, publicado en el Diario Oficial de la Federación el 23 de abril de 2020, según el cual se mandata un recorte presupuestario del $75 \%$ "de las partidas de servicios generales y materiales y suministros" para toda las dependencias y entidades del Gobierno Federal, con excepción de 38 programas prioritarios enlistados en el Acuerdo. 
reguladas en el ordenamiento jurídico mexicano (órdenes de protección, medidas precautorias en materia familiar, medidas de protección en materia penal, pensión alimenticia y convivencia familiar) continuaron inhabilitadas para proteger a las mujeres que se encuentran en situación de violencia durante la contingencia sanitaria (EQUIS, 2020).

Aunado a lo anterior, los Centros de Justicia para las Mujeres -espacios integrales que concentran servicios interinstitucionales y especializados de atención con perspectiva de género para mujeres víctimas de violencia, sus hijas e hijos- cuentan con serios problemas en sus servicios, debido a su falta de normatividad clara y fortaleza institucional, ya que no cuentan con independencia presupuestaria, patrimonio ni personalidad jurídica propia, de acuerdo con reportes de EQUIS: Justicia para las Mujeres (EQUIS, 2020:35; Intersecta ,et al, 2020).

Por su parte, la información pública que permitiría evaluar el impacto que las medidas de confinamiento y el contexto de crisis han tenido en la violencia contra las mujeres, ha resultado insuficiente, poco oportuna y se ha visto limitada por las medidas sanitarias. La Comisión Interamericana de Mujeres ha mostrado que la ausencia de datos desagregados por sexo/ género obstaculiza la toma de decisiones acertadas, por lo que ha llamado a los países de la Región Latinoamericana a generar datos desagregados por sexo y con un enfoque de interseccionalidad de: I) las tasas diferenciadas de infección del coronavirus, 2) los impactos económicos y en la carga diferenciada de cuidados, 3 ) las barreras de acceso de mujeres a servicios básicos de salud y tecnología y 4) las tasas de incidencia de violencias familiar, sexual, acoso, laboral, feminicida, entre otras (OEA, 2020).

En cambio, en México, el Instituto Nacional de Transparencia, Acceso a la Información y Protección de Datos Personales determinó la suspensión de plazos para todos los procesos relacionados con solicitudes de acceso a la información y protección de datos personales. Dicha suspensión permitió a los sujetos obligados a no cumplir con sus obligaciones en materia de transparencia para la entrega de información pública y amplió la fecha hasta el 30 de mayo, dejando así a la ciudadanía imposibilitada para impugnar la falta de información pública (Intersecta, et al, 2020: 47).

Por otro lado, periódicamente el Secretariado Ejecutivo del Sistema Nacional de Seguridad Pública y el Instituto Nacional de Estadística y Geografía ofrecen datos oficiales que permiten conocer información sobre la violencia que experimentan las mujeres dentro y fuera de sus hogares, así como las cifras sobre investigaciones penales abiertas por los delitos de violencia familiar, homicidio y feminicidio, y las llamadas realizadas al número de emergencia 9l I relacionadas con violencia contra las mujeres, o sobre dónde ocurren los homicidios de las mujeres (por ejemplo, si es en la vivienda o no) y si los asesinatos ocurrieron, o no, en un contexto de 
violencia intrafamiliar (Intersecta, et al, 2020; SESNSP, 2020). No obstante, dichas fuentes tienen carencias que impiden conocer con detalle y rapidez cómo afecta la violencia a las mujeres, lo que dificulta identificar otras posibles explicaciones de por qué ha incrementado la violencia hacia este sector de la población.

\section{CONSIDERACIONES FINALES}

La pandemia de COVID-19 nos está poniendo a prueba de maneras que la mayoría de las personas nunca habíamos experimentado con anterioridad (ONU, 2020). El confinamiento sanitario provoca tensiones emocionales, familiares, económicas y laborales que deberemos enfrentar, pero el costo de esta crisis sanitaria no debe recaer de manera desproporcionada en las mujeres. En particular, el incremento de las violencias contra las mujeres era un problema creciente en los últimos años y, en el contexto de la crisis se exacerbó a niveles que hacen posible hablar de dos pandemias que continúan matando y enfermando a las mujeres: las violencias contra las mujeres y el coronavirus.

En este artículo se argumentó que el empeño de las instituciones durante y después del confinamiento debe dirigirse a replantear y transformar la manera en que nos relacionamos hombres y mujeres, impulsando políticas públicas orientadas a crear dinámicas igualitarias y libres de violencia en el espacio privado, así como en la esfera pública (laboral, política, económica). Ante la crisis por la COVID-19, las mujeres son las más afectadas, ya que las desigualdades que han enfrentado a lo largo de la historia las ha relegado a seguir cumpliendo con roles establecidos de género, lo que las orilla a trabajar en las labores domésticas. El equilibrar este trabajo no remunerado puede ser un paso para minimizar la brecha de género en el trabajo. Asimismo, los refugios, las líneas de atención y las casas de justicia para las mujeres se deben considerar como un servicio esencial y deben contar con financiamiento programado, así como amplios esfuerzos destinados a mejorar la difusión de su disponibilidad.

La violencia contra las mujeres es hoy el principal obstáculo para su desarrollo y participación en la vida económica, política, social y cultural del país. Las causas de esta violencia son la desigualdad estructural que se sigue reproduciendo tanto en espacios privados como públicos, así como las masculinidades nocivas y conductas machistas aún vigentes en la sociedad. En este tenor, es fundamental involucrar a las mujeres en todas las fases de la respuesta y en la toma de decisiones nacionales y locales para hacer frente a la pandemia, especialmente a grupos de mujeres que perciben un impacto diferenciado de la crisis, como las mujeres trabajadoras del sector sanitario, trabajadoras domésticas y del sector informal, así como mujeres migrantes y refugiadas. 
Finalmente, se argumentó sobre la importancia de que las instituciones gubernamentales diseñen e implementen un protocolo de actuación integral coordinado e intersectorial con enfoque de género, derechos humanos e interculturalidad, para atender los impactos y secuelas que la COVID- I 9 ha dejado y seguirá dejando en la vida de las mujeres mexicanas. En suma, para dar una respuesta completa y eficaz al grave problema que representan las violencias contra las mujeres y niñas, se requiere de una reforma al sistema de procuración e impartición de justicia, que coloque en el centro los derechos humanos a las mujeres y las niñas, así como la profesionalización y capacitación con perspectiva de género a las y los operadores del sistema de justicia, porque los derechos humanos de las mujeres no se suspenden en ninguna crisis, bajo ninguna circunstancia. 


\section{REFERENCIAS BIBLIOGRÁFICAS}

Cerrillo, Shelma. (2020). Denuncias por violencia familiar siguen creciendo. Cuestione, 31 de agosto de 2020. México.

Comisión Nacional de Derechos Humanos. (2020). Análisis, Seguimiento y Monitoreo de la Política Nacional en Materia de Igualdad entre Mujeres y Hombres. CNDH, México, Pp. 1-109.

Encuesta Nacional sobre la Dinámica de las Relaciones en los Hogares (2016). Cuarta Encuesta Nacional sobre la Dinámica de las Relaciones en los Hogares. Instituto Nacional de Estadística y Geografía, México.

EQUIS: Justicia para las Mujeres (2020). Informe "(Des)Protección Judicial en tiempos del COVID-19". México. Disponible en: https://equis.org.mx/ wp-content/uploads/2020/o8/informe-dospandemiasmexico.pdf

Fríes, Lorena y Victoria Hurtado (2010). Estudio de la información sobre la violencia contra la mujer en América Latina y el Caribe. CEPAL, Serie Mujer y Desarrollo, Santiago, marzo de 2010.

Instituto Nacional de las Mujeres (2020). Violencia contra las mujeres. Indicadores básicos en tiempos de pandemia. Gobierno de México. México. Pp. 1-13.

Intersecta (2020). Las dos pandemias. Violencia contra las mujeres en México en el contexto de COVID-19. Equis: Justicia para las Mujeres, Intersecta, Red Nacional de Refugios. México.

Ley General de Acceso de las Mujeres a una Vida Libre de Violencia (2020). Nueva Ley publicada en el Diario Oficial de la Federación el 1 de febrero de 2007. Texto vigente. Última reforma publicada en el Diario Oficial de la Federación el 13 de abril de 2020, Cámara de Diputados, México.

Organización de las Naciones Unidas (2010). Definición de la violencia contra las mujeres y niñas. Centro virtual de Conocimiento para Poner Fin a la Violencia contra las Mujeres y Niñas.

Organización de las Naciones Unidas (2020). COVID-19 y su impacto en la violencia contra las mujeres y niñas. Disponible en https://www2. unwomen.org/-/media/field\%20office\%20mexico/documentos/publicaciones/2020/abril\%202020/covid19_violenciamujeresninas_generalabril2020.pdf?la=es\&vs $=2457$

Organización de los Estados Americanos (2020). COVID-19 en la vida de las mujeres. Razones para reconocer los impactos diferenciados. Comisión Interamericana de Mujeres. Disponible en: http://www.oas.org/es/cim/ docs/ArgumentarioCOVID19-ES.pdf

Secretariado Ejecutivo del Sistema Nacional de Seguridad Pública. (SESNSP, 2020). Información Sobre violencia contra las mujeres. Incidencia delictiva y llamadas de emergencia 9-1-1. Centro Nacional de Información. Corte al 31 de julio de 2020. 
Toche, Nelly. (2020). Embarazos de adolescentes han aumentado 20\% durante la pandemia. En El Economista. México.

Vázquez Correa, Lorena; Patiño Fierro, Martha Patricia. (2020). “Violencia política contra las mujeres y paridad de género: de la presencia en el poder a la transformación de la política" Cuaderno de investigación No. 67. Instituto Belisario Domínguez, Senado de la República, Ciudad de México, P. 78. 